\section{Desafios e estratégias na gestão do processo editorial e divulgação do periódico científico e-Scientia}

Resumo: Esse relato de caso apresenta os desafios e estratégias na gestão do processo editorial e na divulgação do periódico da área da saúde, e-Scientia. Os principais desafios encontrados são a baixa visibilidade do periódico e a designação, comunicação e uso da plataforma OJS pelos autores e revisores. Firmar parceria com Ligas Acadêmicas e organizadores de eventos, via publicação de Anais, melhorou a divulgação do periódico e reduziu as submissões endógenas. A adoção de planilha e a intensificação do uso de e-mails complementaram o OJS no processo de editoração e de comunicação com autores e revisores. Mais estratégias precisam ser adotadas para promover o desenvolvimento da e-Scientia.

Palavras-chave: Editoração. Revisão por pares. Comunicação e Divulgação Científica

Challenges and strategies in managing the editorial process and dissemination of the e-Scientia scientific journal

Abstract: This article presents the challenges and strategies in managing of the editorial process and dissemination of e-Scientia, a Private University's health area journal. The strategy to improve visibility was to establish a partnership with Academic Leagues, publishing Event Annals, thus promoting the journal, which reduced endogenous submissions. The strategy to improve the monitoring of the publishing process, in addition to the OJS, adopted a complementary spreadsheet. To minimize the problems related to the use of the system by the authors, e-mail was adopted as a form of communication. It is observed that the appointment of reviewers is still a challenge to be overcome during the article publication process.

Keywords: Publishing. Peer Review. Scientific Communication and Diffusion.

Retos y estrategias en la gestión del proceso editorial y la difusión de la revista científica e-Scientia

Resumen: El objetivo del trabajo es presentar los desafíos y estrategias en la gestión del proceso editorial y difusión de la revista electrónica eScientia, desde una Institución Privada de Educación Superior. La estrategia para mejorar la visibilidad fue establecer una alianza con Ligas Academicas, publicando Anales de eventos, promocionando la revista y reducindo las presentaciones endógenas. La estrategia para mejorar el seguimiento del proceso de publicación, además del OJS, se adoptó de una hoja de cálculo complementaria. Para minimizar los problemas con el uso del sistema por parte de los autores, se adoptó el correo electrónico como forma de comunicación. Se observa que la designación de revisores sigue siendo un desafío a superar durante el proceso de publicación del artículo.

Palavras Clave: Edición. Revisión por Pares. Comunicación y Divulgación Científica.
Cláudia Lopes Penaforte

Belo Horizonte, Minas

Gerais, Brasil

Professora do Centro

Universitário de Belo

Horizonte, UNIBH e

Faminas $\mathrm{BH}$

\section{Silvia Q. Savergnini}

Belo Horizonte, Minas

Gerais, Brasil.

Professora do Centro

Universitário de Belo

Horizonte, UNIBH e da

Universidade do Estado de Minas Gerais, UEMG.

\section{Nathan Mendes Souza}

Belo Horizonte, Minas

Gerais, Brasil.

Professor, Faculdade de

Medicina, Universidade

Federal de Minas Gerais.

Membro, Comissão

Assessora de Avaliação

da Formação Média,

Instituto Nacional de

Estudos e Pesquisas

Educacionais Anísio

Teixeira/MEC. 


\section{Introdução}

A publicação de um manuscrito é uma forma de disseminar conhecimentos relevantes e confiáveis à comunidade científica. Assim, o periódico científico pode aproximar cientistas, gestores e sociedade civil e auxiliar no processo de tomada de decisões.

Os periódicos eletrônicos apresentam vantagens como: acesso remoto de baixo custo, rápida circulação da informação, fácil localização via uso de mecanismos de buscas e eficiente editoração do periódico. Esses periódicos contam com plataformas profissionais para a gestão da editoração, mas a falta de familiaridade com as plataformas é um desafio para os usuários. Outros desafios na editoração de periódicos incluem a insuficiência de recursos humanos e a visibilidade do periódico.

O presente trabalho objetiva apresentar os desafios e estratégias na gestão do processo editorial e de divulgação da e-Scientia, um periódico eletrônico da área da saúde de uma Instituição Privada de Ensino Superior.

\section{Metodologia}

Trata-se de um relato de caso, onde apresenta-se os desafios e estratégias na gestão do processo editorial e da divulgação do periódico da área da saúde, e-Scientia (ISSN 1984-7688), mantido por uma Instituição privada de Ensino Superior.

O periódico e-Scientia é multidisciplinar, em formato eletrônico, mantido desde 2008 pelo Centro Universitário de Belo Horizonte (UniBH), Minas Gerais, instituição pertencente ao grupo Ânima Educação. O UniBH abriga vários cursos de ensino superior na área da saúde e possui uma vertical Nacional/Ânima do curso de Medicina, a Inspirali. O periódico disponibiliza, semestralmente, todo o seu conteúdo de forma gratuita pelo site https://revistas.unibh.br/dcbas. Possui indexação em Latindex, Diadorim, Sumarios.org, Google acadêmico, Periódicos de Minas e, na avaliação do Qualis/CAPES (2013-2016), apresenta a classificação B5 na área de Saúde Coletiva. A submissão de trabalhos é gratuita, por fluxo contínuo, pelo portal do periódico, que abriga publicações de artigos originais, revisão sistemática e integrativa, relato de caso e metanálise. As diretrizes para os autores são disponibilizadas na plataforma e segue parâmetros nacionais sobre a ética em pesquisa de acordo com o Conselho Nacional de Ética em pesquisa (CONEP) e a Comissão de ética em uso de animais (CEUA). 
O sistema de gestão do periódico é o Open Journal System (OJS)/Serviço de Editoração Eletrônico de Revistas (SEER). Adota formato de preservação digital Rede Cariniana IBICT e Lots of Copies Keep Stuff Safe (LOCKSS) e utiliza o recurso de detecção de similaridade plágio o Plagiarism Check.

Os trabalhos submetidos ao periódico são avaliados por dois membros do Conselho Científico/Avaliador ou ad hoc, em formato de avaliação às cegas. No cadastro dos avaliadores é solicitado o registro Open Researcher and Contributor ID (ORCID), e a avaliação dos manuscritos pode ser realizada através do SEER, utilizando um formulário semiestruturado ou, se desejar, o avaliador poderá utilizar um roteiro de avaliação.

\section{Resultados e discussão}

Desafios e estratégias desenvolvidas para melhoria do Processo de Editoração do periódico

Todas as etapas de editoração são de responsabilidade da equipe de editorial do e-Scientia, que utiliza a plataforma SEER para gestão. Embora a ferramenta SEER seja, em geral, suficiente para a gestão do processo, a falta de familiaridade com a plataforma levou ao desenvolvimento de estratégias para controle paralelo. Uma estratégia adotada foi a criação de uma planilha, onde são registradas todas as etapas, desde a submissão até a publicação do artigo, o que permite o acompanhamento do fluxo de cada processo. Para minimizar os problemas relacionados ao uso do sistema pelos usuários/autores/revisores, intensificou-se uso de e-mails para melhoria da comunicação.

Observou-se também um não retorno, em tempo hábil, da versão alterada pelos autores após as sugestões dos pareceristas. Ao submeter o manuscrito no sistema, registrava-se os dados de apenas um dos autores, o que dificultava a comunicação, pois nem sempre esse autor está atento às solicitações do periódico. Uma estratégia utilizada foi ressaltar, no documento "Normas para preparação e submissão", a necessidade de realizar o cadastro completo no sistema, de todos os autores do manuscrito.

\section{Desafios e estratégias desenvolvidas para melhoria da divulgação do periódico}

Uma estratégia adotada pelo corpo editorial, para melhoria da visibilidade da eScientia, foi firmar parcerias com Ligas Acadêmicas de cursos da saúde de várias instituições. O periódico tem publicado, na forma de resumo expandido, os Anais dos Eventos promovidos pelas Ligas. No segundo semestre de 2020, o periódico publicou os Anais de três eventos, onde cerca de $60 \%$ dos resumos pertenciam a outros Estado brasileiros. Essa estratégia gerou um aumento na submissão de manuscritos de outros 
Estados, demonstrando uma melhoria na visibilidade do periódico e uma diminuição da endogenia nas publicações.

\section{Desafios futuros}

O tempo de revisão dos manuscritos quanto à conformidade desk review, para avaliação em relação ao escopo do periódico é de cerca de quinze dias. O tempo da avaliação por pares tem sido, em média, de um mês. O corpo editorial faz a análise dos pareceres e comunicação aos autores, que tem cerca de 20 dias para retornarem 0 manuscrito com as adequações. O manuscrito corrigido é enviado aos avaliadores, que retornam em aproximadamente, 20 dias. Se aprovado, a equipe editorial realiza a normalização, revisão de metadados, diagramação, edição de layout, leitura da prova aos autores, finalização do artigo e divulgação. O tempo de submissão à publicação de um manuscrito tem sido, em média, de 90 a 120 dias.

O processo de revisão dos manuscritos adotado pela e-Scientia é através da revisão por pares e às cegas. Segundo Barata (2019), esse processo traz dificuldades para a editoração das revistas como: recusa em revisar, revisão em tempo hábil, qualidade da revisão e preparação dos revisores, excesso de manuscritos para revisão, conflitos de interesse econômicos e não econômicos. $\mathrm{O}$ UniBH, além da e-Scientia, mantém outros quatro periódicos nas áreas de direito, humanas, sociais e exatas, que utilizam a mesma plataforma de gestão e contam com um único banco de cadastro de avaliadores, sem distinção de áreas de atuação. Muitas vezes o cadastro dos avaliadores encontra-se desatualizado, incompleto ou incorreto, tornando esse avaliador inelegível. Para minimizar o problema, a e-Scientia, tem solicitado aos avaliadores que mantenham o cadastro atualizado e preencham o campo "área de atuação".

Todos esses entraves aumentam o prazo entre a submissão, avaliação e publicação de um manuscrito pré-selecionado. Outras propostas precisam ser consideradas, incluindo ampliação e mescla intergeracional do corpo editorial (DEEMER, et al., 2021), ensino sobre produção e ciclo de publicação de artigos na graduação dos cursos da saúde (SERRA, 2020) e implementação de vídeos tutoriais para o uso da plataforma OJS (ORTEGA et al., 2019), o que poderia ser viabilizado com maior apoio institucional, trabalho colaborativo em equipe e capacidade ampliada de gestão.

\section{Considerações finais}

Este relato evidencia alguns desafios e estratégias utilizadas pelo corpo editorial da e-Scientia para ser reconhecido como um periódico de rigor metodológico e científico. 
Soluções como aumento de parcerias com Ligas Acadêmicas, uso de planilhas complementares com prazos e acompanhamento de todo o processo, tem contribuído com a agilidade e visibilidade da e-Scientia.

\title{
Referências
}

ORTEGA, L. A. et al. Open Journal Systems: resultados de la implementación de videos tutoriales como iniciativa para el proceso editorial de una revista. MEDISAN. Santiago de Cuba, v. 23, n. 6, p. 1104-1113, 2019. Disponível em: http://scielo.sld.cu/scielo.php script=sci_arttext\&pid=S1029-30192019000601104\&lng=es\&nrm=iso. Acesso em: 01 jul. 2021.

BARATA, R. B. Desafios da editoração de revistas científicas brasileiras da área da saúde. Ciência \& Saúde Coletiva. v. 24, n. 3, p. 929-939, 2019. Disponível em: https://doi.org/10.1590/1413-81232018243.29952016. Acesso em: 26 jun. 2021.

DEEMER, B. R. et al., Engaging the next generation of editorial talent through a hands-on fellowship model. Ecology Letters. v. 24, p. 1297-130, 2021. Disponível em: https://doi.org /10.1111/ele.13758. Acesso em: 01 jul. 2021.

SERRA, M. E. Gestión de un artículo científico. ¿Cómo llega lo que leemos a las revistas médicas? Arch. Argent. Pediatr. v. 118, n. 6, p. 433-437, 2020. Disponível em: https://www.sap.org.ar/docs/publicaciones/archivosarg/2020/v118n6a14.pdf. Acesso em: 01 jul. 2021.

\author{
Cláudia Lopes Penaforte \\ Belo Horizonte, Minas Gerais, Brasil. \\ Professora do Centro Universitário de Belo Horizonte- UniBH \\ Professora da Faminas $\mathrm{BH}$
}

Minicurrículo: Doutora em Bioquímica pelo Programa de Pós-Graduação em Bioquímica e Imunologia da Universidade Federal de Minas Gerais. Mestra em Bioquímica e Imunologia pelo Programa de Pós- Pós-Graduação em Bioquímica e Imunologia da Universidade Federal de Minas Gerais. Editora do Periódico eScientia.

Contribuição de autoria: Realizou a escrita, curadoria de dados, a metodologia, copilou os resultados e discussão, revisão e edição, validação.

ORCID ID: https://orcid.org/0000-0002-0337-0272

Lattes: http://lattes.cnpq.br/8368159326859315.

E-mail: claudiapenaforte@gmail.com 


\section{Silvia Silveira Quintão Savergnini}

Belo Horizonte, Minas Gerais, Brasil.

Professora do Centro Universitário de Belo Horizonte- UniBH

Professora da Universidade do Estado de Minas Gerais - UEMG

Minicurrículo: Mestre e Doutora em Fisiologia e Farmacologia pela Universidade Federal de Minas Gerais/UFMG, pós-doutorado pelo Swiss Federal Institute of Technology/EPFL - Suíça e pela UFMG em Fisiologia e Farmacologia.

Contribuição de autoria: Análise Formal, Revisão e Edição, Validação.

ORCID ID: https://orcid.org/0000-0002-3542-0174

Lattes: http://lattes.cnpq.br/9444858785437053

E-mail: silviaquintao@gmail.com

\section{Nathan Mendes Souza}

Belo Horizonte, Minas Gerais, Brasil.

Professor, Faculdade de Medicina, Universidade Federal de Minas Gerais.

Membro, Comissão Assessora de Avaliação da Formação Médica, Instituto Nacional de Estudos e Pesquisas Educacionais Anísio Teixeira/MEC.

Minicurrículo: Médico de Família e Comunidade, mestre em educação médica (UFOP) e doutorando em Metodologias de Pesquisa em Saúde (McMaster University).

Contribuição de autoria: Realizou a administração do projeto, Análise Formal, Revisão e Edição, Metodologia e Validação e Visualização.

ORCID ID: https://orcid.org/0000-0002-4341-1964

Lattes: http://lattes.cnpq.br/3667197759813675

E-mail: nathanmendes@hotmail.com 